\title{
Une nationalité à construire : le cinéma brésilien et la recherche de l'unité nationale dans l'Institut national de cinéma éducatif (INCE)
}

A Nationality to Build: Brazilian Cinema and the Search for the Unity of the Nation in the National Institute of Educational Cinema

\section{Rafael Beverari}

\section{OpenEdition \\ Journals}

Édition électronique

URL : https://journals.openedition.org/itti/2503

DOI : 10.4000/itti.2503

Éditeur

Université de Poitiers

Référence électronique

Rafael Beverari, «Une nationalité à construire : le cinéma brésilien et la recherche de l'unité nationale dans I'Institut national de cinéma éducatif (INCE) », Images du travail, travail des images [En ligne], 12 I 2022, mis en ligne le 16 février 2022, consulté le 02 avril 2022. URL : http://journals.openedition.org/ itti/2503 ; DOI : https://doi.org/10.4000/itti.2503

Ce document a été généré automatiquement le 2 avril 2022.

Images du travail, travail des images 


\title{
Une nationalité à construire : le cinéma brésilien et la recherche de l'unité nationale dans l'Institut national de cinéma éducatif (INCE)
}

\author{
A Nationality to Build: Brazilian Cinema and the Search for the Unity of the \\ Nation in the National Institute of Educational Cinema
}

Rafael Beverari

1 Cet article présente les enjeux d'une recherche intitulée « Investir pour progresser? Le concept du travail dans la production audiovisuelle de l'Institut national de cinéma éducatif $»^{1}$. Il cherche à comprendre comment les représentations du travail se retrouvent dans le matériel filmique produit par cet institut - considéré comme le premier organe spécifiquement axé sur le secteur audiovisuel brésilien, ayant existé entre les années 1936 et 1966. L'analyse part de la production cinématographique pour arriver à la compréhension d'un contexte social et politique qui repose sur la consolidation d'une classe ouvrière en constante transformation.

2 Le corpus filmique ne s'est pas limité au choix d'un certain genre cinématographique, comme la fiction ou le documentaire - tous deux présents dans la collection. Le thème de travail apparaît comme transversal face aux autres sujets traités, tels que la diffusion scientifique, les biographies de célébrités, les caricatures, les événements commémoratifs et folkloriques, la géographie des villes, etc. Le contenu des productions a été analysé en tenant compte de la structure organisationnelle qui l'a produit (INCE) et de son contexte historique. En partant de la compréhension de l'organisation des forces productives, nous cherchons à étudier comment la conception du travail se construit dans les scènes, et ceci en portant un regard attentif aux travailleurs dans ce processus. En ce sens, le positionnement et le déplacement de la caméra, le processus de montage, les effets sonores et la voix hors champ du narrateur sont des indicateurs intéressants. 
3 La collection de l'INCE se trouve actuellement à la Cinémathèque brésilienne. Sur un total de 407 films réalisés par l'Institut national de cinéma éducatif, seuls 218 peuvent être visionnés. Parmi les 189 films restants de la collection, certains n'ont pas été retrouvés et d'autres sont dans un état de détérioration qui rend leur visionnement impossible. La partie disponible pour la recherche sur la documentation écrite fait partie des archives de Gustavo Capanema, créateur de l'INCE et ministre de l'Éducation et de la Santé publique de 1934 à 1945. Une autre source de documents utilisée est la collection d'Edgard Roquette-Pinto, un médecin, professeur et anthropologue brésilien renommé qui a dirigé l'INCE pendant ses onze premières années (1936-1947). Dans cette collection, nous trouvons une série intitulée «Cinema Educativo » avec des lettres et des communications de Roquette-Pinto concernant la cinématographie éducative.

Tableau 1 : Composition du matériel cinématographique produit par l'Institut national de cinéma éducatif

\begin{tabular}{|l|l|l|}
\hline \multicolumn{2}{|l|}{ Catalogue des films de l'INCE } \\
\hline Année & $\begin{array}{l}\text { Productions } \\
\text { audiovisuelles }\end{array}$ & $\begin{array}{l}\text { Des films qui abordent } \\
\text { la question du travail }\end{array}$ \\
\hline 1936 & 30 & 9 \\
\hline 1937 & 34 & 8 \\
\hline 1938 & 28 & 12 \\
\hline 1939 & 42 & 11 \\
\hline 1940 & 20 & 7 \\
\hline 1941 & 15 & 5 \\
\hline 1942 & 25 & 12 \\
\hline 1943 & 13 & 5 \\
\hline 1944 & 15 & 6 \\
\hline 1945 & 11 & 7 \\
\hline 1946 & 8 & 7 \\
\hline 1947 & 11 & 7 \\
\hline 1948 & 13 & 7 \\
\hline 1949 & 8 & 7 \\
\hline 1950 & 7 & 7 \\
\hline 1951 & 8 & 7 \\
\hline
\end{tabular}




\begin{tabular}{|c|c|c|}
\hline 1952 & 8 & 6 \\
\hline 1953 & 8 & 4 \\
\hline 1954 & 10 & 6 \\
\hline 1955 & 7 & 4 \\
\hline 1956 & 7 & 3 \\
\hline 1957 & 5 & 2 \\
\hline 1958 & 9 & 5 \\
\hline 1959 & 4 & 2 \\
\hline 1960 & 7 & 4 \\
\hline 1961 & 1 & 1 \\
\hline 1962 & 13 & 6 \\
\hline 1963 & 2 & 2 \\
\hline 1964 & 11 & 5 \\
\hline 1965 & 3 & 1 \\
\hline 1966 & 23 & 8 \\
\hline $\begin{array}{l}\text { Non } \\
\text { daté }\end{array}$ & 1 & 1 \\
\hline & 407 & 174 \\
\hline
\end{tabular}

Sources : catalogue préparé par Souza (1990).

4 On observe qu'environ $43 \%$ des films traitent du thème du travail au Brésil. Cette classification a été faite après avoir consulté les fiches techniques des films et visionné le matériel disponible. Bien que certains titres ne fassent pas immédiatement référence à l'objet analysé, il est possible de voir comment le thème du travail est transversal à divers sujets. Ainsi, une production intitulée, par exemple, Ville de São Paulo (1949) peut apporter les preuves de la représentation des travailleurs dans certains quartiers de cette municipalité ; ou encore le film Hygiène rurale (1954) qui, bien que traitant d'une question de santé, fournit des informations sur les conditions de vie des travailleurs ruraux à cette époque. 


\section{L'accent mis sur le travail et la formation professionnelle}

5 Le début du $\mathrm{xx}^{\mathrm{e}}$ siècle est marqué par une large diffusion du cinéma éducatif dans le monde, grâce aux efforts notamment de l'Institut international de cinématographie éducative (ICE). Créé en 1927 à l'initiative de la Commission internationale de coopération intellectuelle, un organe de la Société des Nations, l'Institut a mené ses activités jusqu'en 1937. Parmi les points du statut organique de l'ICE, il est souligné que «l'objectif de l'Institut est de promouvoir la production, la distribution et l'échange entre les différents pays de films éducatifs concernant l'instruction, l'art, l'industrie, l'agriculture, le commerce, l'hygiène, l'éducation sociale, etc. » (Taillibert, 1999, 83).

6 Les rapprochements entre le Brésil et l'ICE sont enregistrés depuis 1929, lorsque Luciano de Feo, alors directeur, a invité Edgar Roquette et Jonathas Serrano (professeur et critique de cinéma) à écrire pour le magazine de cette institution internationale. Dans son invitation aux Brésiliens, le représentant souligne :

les domaines de notre activité sont très vastes. De l'hygiène, de la prévision sociale dans ses différents aspects, aux problèmes du travail comme organisation et rationalisation, à l'agriculture, aux problèmes sociaux [...] $]^{2}$.

7 En octobre 1930, Serrano écrit un article pour le magazine détaillant l'exposition cinématographique éducative qui avait eu lieu au Brésil en 1929.

8 À la suite de ces événements mondiaux, le débat sur l'organisation du cinéma national est imprégné des différents intérêts des classes dominantes présentes sur l'ensemble du territoire brésilien au début du $\mathrm{xx}^{\mathrm{e}}$ siècle. La création de l'Institut national de cinéma éducatif (INCE) en 1936 intervient dans une période marquée par des incertitudes quant à la consolidation d'une idéologie nationaliste et modernisatrice qui est présente, non sans une lutte avec la classe ouvrière, dans la mise en œuvre de différentes politiques publiques. Ainsi, la communication, et notamment le cinéma, est un instrument important pour la diffusion de ces idées.

9 Le principal moyen trouvé par la force politique émergente brésilienne, issue des militaires, des industriels et du milieu artistique des années 1920, pour faire face aux problèmes de leur temps est l'appel au nationalisme. Il n'est donc pas surprenant que des intellectuels participent à la production d'idéaux nationalistes dans le but d'unifier un pays de taille continentale comme le Brésil. En ce moment de consolidation des forces sur le territoire national, la participation des nouveaux managers suit également ce même chemin dans la construction d'une communauté structurée à partir de ses mythes d'origine, de sa langue, de ses vieux documents et autres événements d'un passé qui renforce le sentiment d'unité. Après tout, « plus qu'inventées, les nations sont imaginées, au sens où elles font sens pour l'âme et constituent des objets de désirs et de projections » (Anderson, 2008, 10).

10 Puisque l'un des piliers de la crédibilité d'un discours est de ne pas laisser transparaître la construction qui le soutient, la question qui imprègne ce moment est de savoir quels intérêts sert une telle demande de réalisation de films. Dans un pays dont le taux d'analphabétisme atteint $65 \%$ en 1920 et $56 \%$ en 1940, l'utilisation des images a une fonction importante pour la diffusion de l'information.

11 Car c'est à la recherche du contrôle de ce moyen d'expression que, dans un discours de 1934, le président Getúlio Vargas $(1938,187)$ vante le cinéma comme l’un des «acteurs 
d'instruction les plus utiles dont dispose l'État moderne». De cette façon, la cinématographie rapprocherait " par la vision incisive des faits, les différents noyaux humains, dispersés dans le vaste territoire de la République » (Ibid.). Il ajoute :

Ainsi, comme élément de culture, influençant directement le raisonnement et l'imagination, elle affine les qualités d'observation, accroît les connaissances scientifiques et divulgue la connaissance des choses, sans exiger l'effort et les réserves d'érudition que le livre demande et que les professeurs, dans leurs classes, exigent ${ }^{3}$.

C'est dans ce contexte que le décret $\mathrm{n}^{\circ} 21.240$ de 1932 stipule que "seront considérés comme éducatifs, au jugement de la commission, non seulement les films dont le but est de divulguer des connaissances scientifiques", mais aussi «ceux dont les intermèdes musicaux ou figuratifs se développent autour de motifs artistiques, tendant à révéler au public les grands aspects de la nature ou de la culture ». L'INCE suit ces préceptes en ce qui concerne le "cinéma éducatif ». Dans ces moules, tout thème de film devrait passer par le tamis du ministère de l'Éducation et de la Santé publique. Cette institution, qui traitait des questions d'éducation et de santé dans le même cadre, était également chargée de gérer l'INCE.

13 Après des années de débats sur la nécessité de créer une agence de systématisation de l'audiovisuel, l'Institut national du cinéma éducatif a finalement été réglementé en 1937 - mais en activité depuis 1936. L'INCE présente des thèmes éducatifs aux formes les plus variées avec peu d'apparitions des hommes d'État. Les découvertes scientifiques, le processus d'alphabétisation, les cultures locales, les fables, les hommages aux artistes, les processus de fabrication et l'organisation des forces productives sont quelques-uns des thèmes abordés dans les œuvres. C'est-à-dire, la trajectoire $\mathrm{du}$ processus de rationalisation du travail au Brésil traverse les photogrammes du cinéma national. Les films de l'INCE ont été montrés dans des institutions culturelles, des écoles et, avant les longs métrages, dans les salles de cinéma.

14 Ces productions partent de l'hypothèse de la « nécessité de présenter le cinéma comme étant une expression du réel et de masquer constamment qu'il est artifice, manipulation, interprétation » (Bernardet, 1980, 10). Toutefois, il est intéressant d'y observer comment « le thème du travail horizontalise les approches du milieu rural et des hommes, des femmes et des enfants» (Rangel, 2010, 34) dans les œuvres produites par l'INCE.

15 Le film A Balata - l'un des premiers films distribués par l'INCE en 1936 - est un exemple de cette frontière ténue entre le cinéma et le travail. L'écran noir est composé des titres en blanc A Balata et Amazonas. Un homme noir au centre de l'écran se tient de profil, tenant une corde. Par la surimpression, il est remplacé par une scène en gros plan de ce qui serait une chaussure munie de crampons. Avec des outils de travail correctement présentés au public, un travailleur commence à faire des coupes diagonales dans le tronc d'un arbre. Un plan général permet de voir la poursuite de son travail de coupe intense. Une autre surimpression fait référence à un très gros plan. Les instruments de travail, indiqués dans les premières scènes, sont soulignés devant une prise de vue en contre-plongée qui met en évidence les gestes du travailleur dans une perspective qui donne la priorité au personnage représenté. Les équipements de sécurité se résument à ceux pointés au début de la scène, à savoir une corde et une chaussure avec des crampons (image 1). Une scène curieuse attire l'attention à la fin du film lorsqu'un travailleur allume une cigarette dans la bouche d'un autre. Les deux travailleurs noirs 
fument en face du liquide blanc qui les attend pour être manufacturé (image 2). C'est ainsi que le travailleur du caoutchouc est présenté sur les écrans.

Image 1 : Extrait du film A Balata présentant le travailleur avec ses mauvais outils de travail

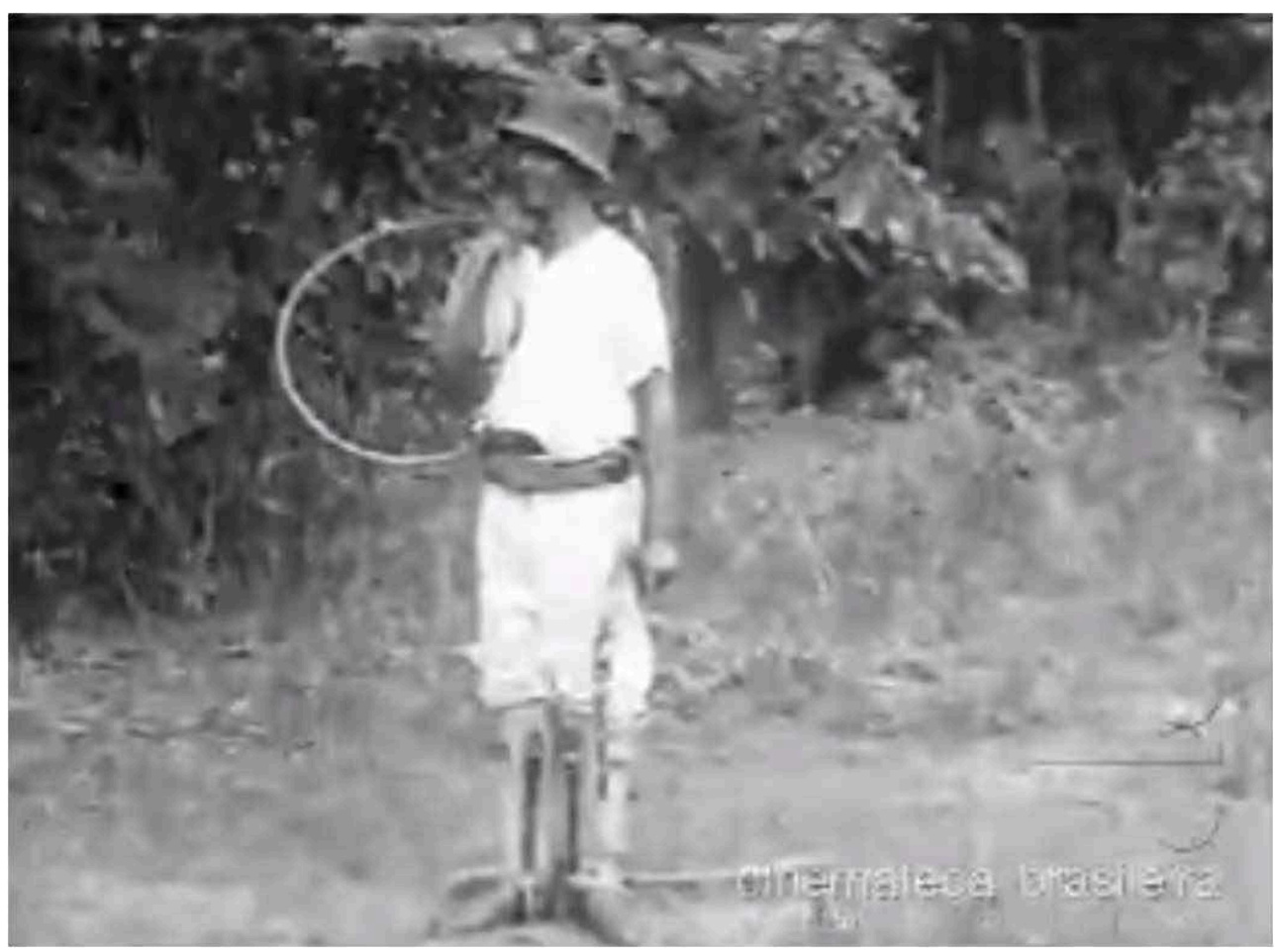

A Balata, 1936, Agesilau de Araujo et Silvino Santos, coll. Cinemateca Brasileira. 
Image 2 : Extrait du film $A$ Balata qui présente les travailleurs confrontés à des conditions de travail précaires à l'intérieur des forêts

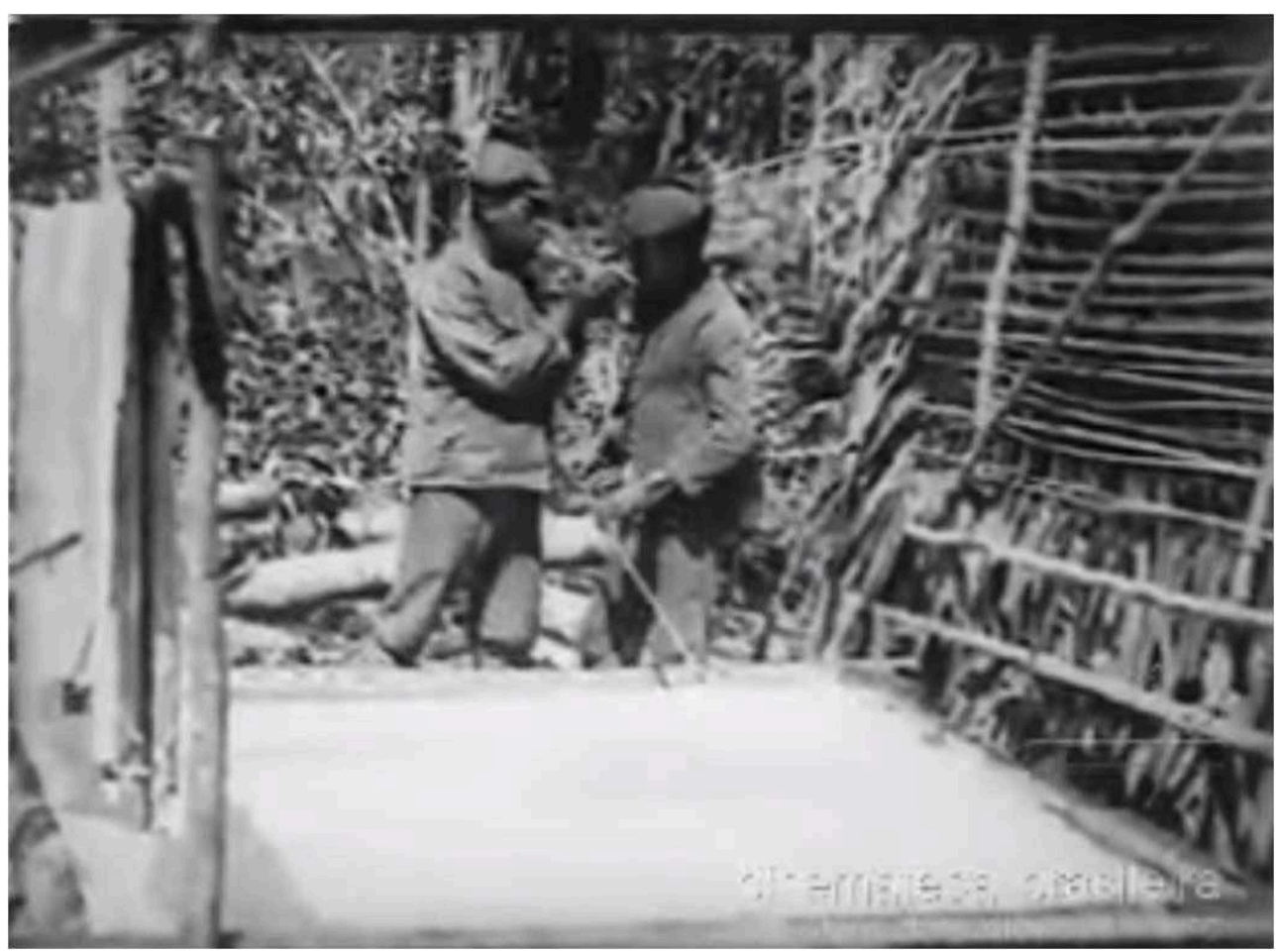

A Balata, 1936, Agesilau de Araujo et Silvino Santos, coll. Cinemateca Brasileira.

Une autre œuvre audiovisuelle sur la production d'une marchandise est Assemblage $d u$ moteur, réalisée en 1942 par Oscar Motta Vianna da Silva. Loin du processus de manufacture présenté dans la première vidéo, ce court métrage montre le processus de fabrication des moteurs dans la municipalité de Rio de Janeiro. La complexité des activités est mise en évidence par la voix hors-champ qui relate que « dans une usine, des ouvriers enlèvent à coups de marteau les billes latérales de l'arbre du rotor d'un moteur qui est ensuite doté d'une base filetée dans son propre corps. Les fils conducteurs sont enroulés autour de l'arbre du rotor, fixés uniformément à l'aide d'un marteau, puis isolés... ». De cette façon, Maurício Tragtenberg $(1974,71)$ précise avec ironie que :

chaque opération est décomposée en temps élémentaires, avec l'aide du chronomètre. Taylor détermine le temps moyen pour chaque élément de base du travail, en agrégeant les temps élémentaires et les temps morts, pour obtenir le temps total du travail, dans le but messianique d'éviter le plus grand péché de tous - la perte de temps.

Les gros plans montrent la minutie des activités effectuées par les ouvriers pendant la production (image 3). Contrairement au dernier court métrage analysé, en suivant le rythme des machines, les travailleurs n'apparaissent pas comme des fumeurs. 
Image 3 : Extrait du film Assemblage du moteur qui présente le travail face aux machines

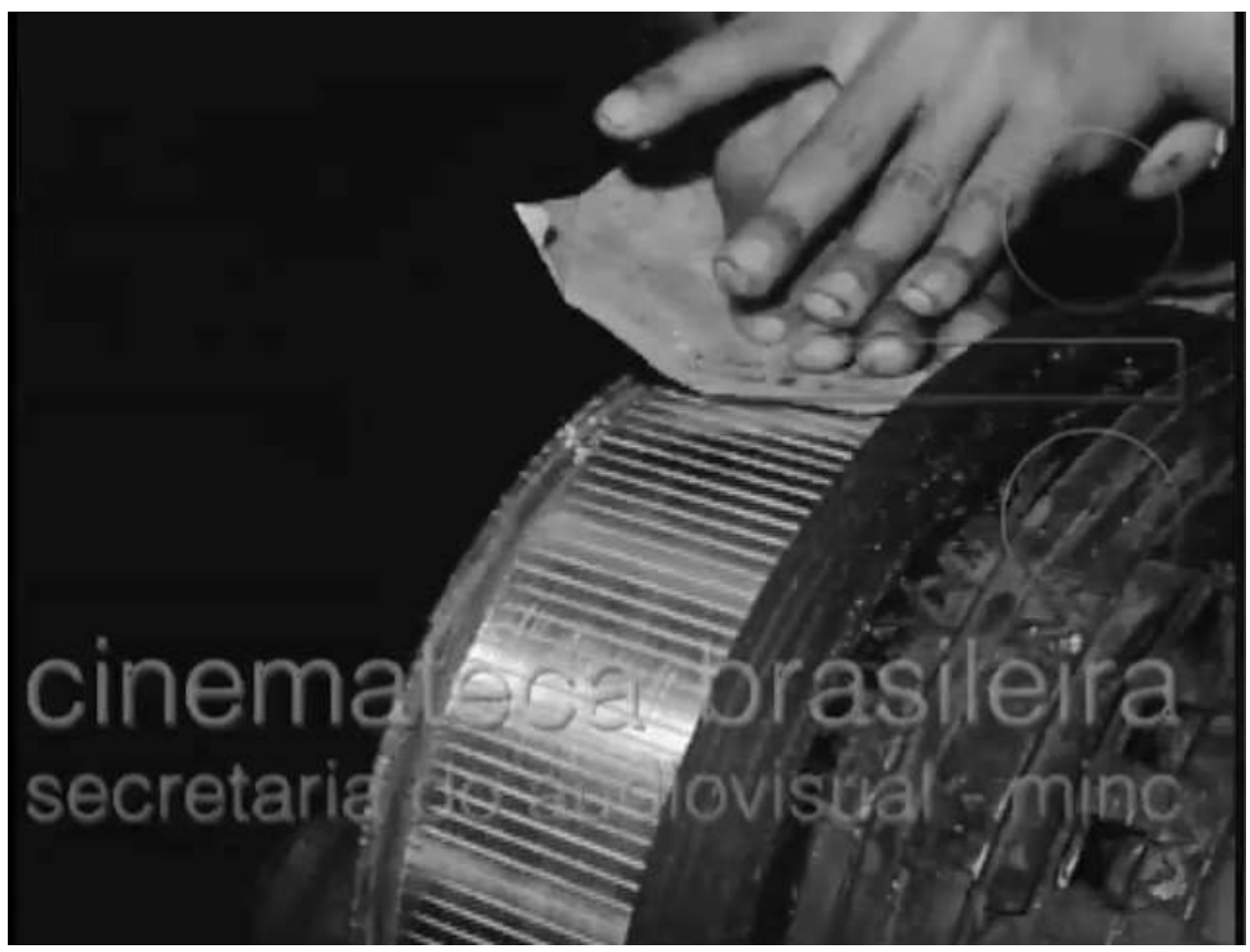

Montagem de motor, 1942, Oscar Motta Vianna da Silva, coll. Cinemateca Brasileira.

Cette extrême spécialisation des fonctions exercées par la main-d'œuvre peut également être observée dans la production de 1945 de Humberto Mauro intitulée L'Enseignement industriel au Brésil. Les bâtiments de l'école technique de Vitória, apparaissent sur le chemin des jeunes étudiants. Alors que les images de femmes (image 4) indiquent le cours, selon le narrateur, de « coupe et de couture », le tournage, le pressage, le classement mécanique et le classement manuel sont représentés par des étudiants masculins. Cependant, la voix hors champ complète que « la loi organique de l'enseignement industriel n'établit pas d'exclusivité de sexe pour aucune des activités de son atelier. Elle ne fait que l'adapter aux possibilités naturelles ». Ainsi, la division sexuelle du travail fait irruption face à cette séparation des activités. À ce stade, il convient de noter, selon Aurélie Jeantet $(2012,9)$, que « les films sont porteurs de représentations sociales qui véhiculent des stéréotypes, des idées, des normes, des valeurs et des idéologies ». 
Image 4 : Extrait du film L'Enseignement industriel au Brésil et la question de la division sexuelle du travail

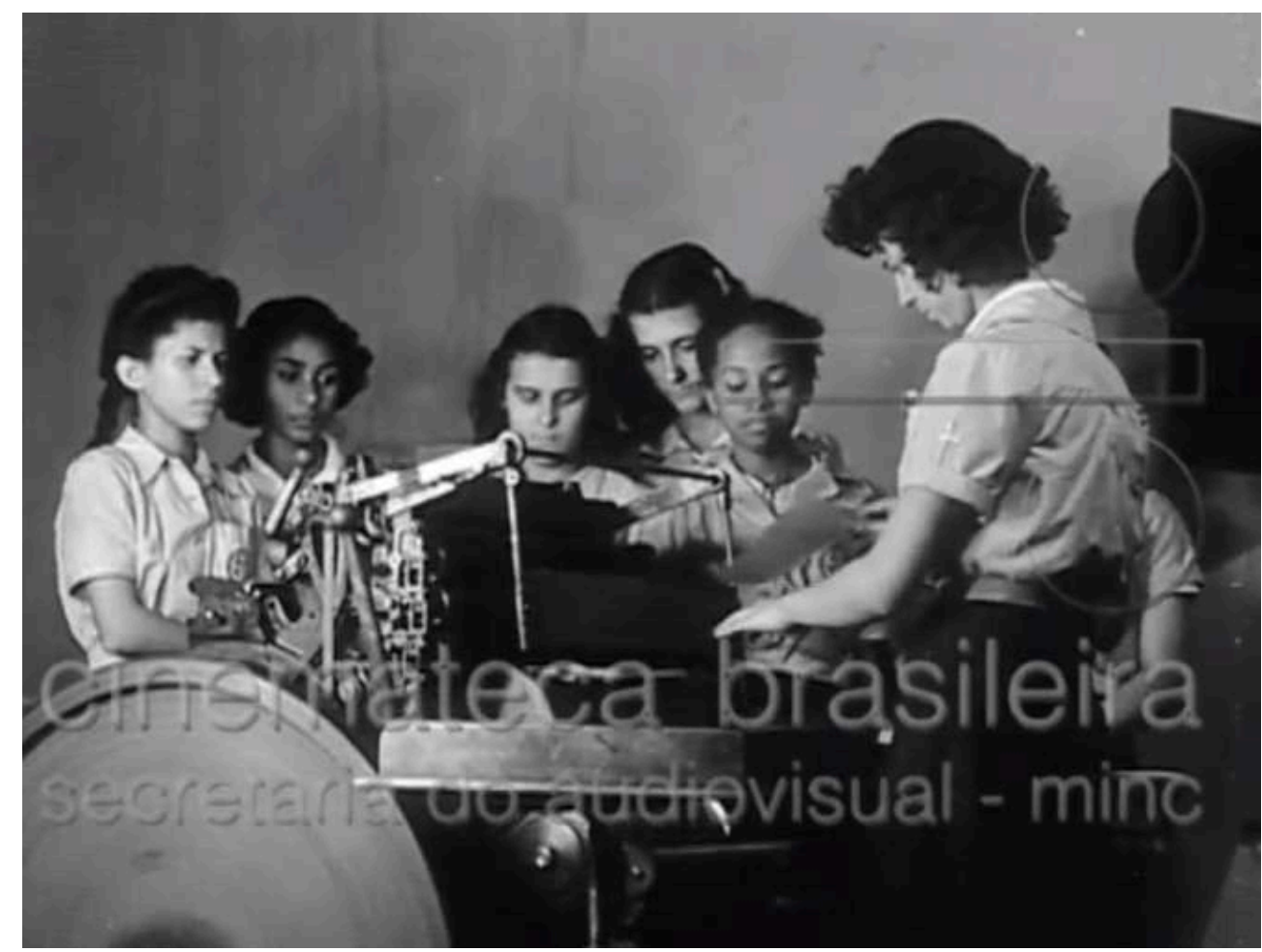

O ensino industrial no Brasil, 1945, Humberto Mauro, coll. Cinemateca Brasileira.

\section{Une volonté de promouvoir le progrès technologique}

Dans le sillage de la formation des travailleurs, le court métrage de 1958, Éducation et Développement, relate la construction de 48 écoles. Une scène dans laquelle un homme en tracteur et un homme à cheval (image 5) sont placés face à face attire l'attention. Puis le narrateur affirme que "sans le minimum d'instruction, désarmé par la mécanisation de l'agriculture, [l'homme] n'obtient rien ». La saga se poursuit en ville alors que le personnage cherche un emploi dans une usine de textile. Bientôt, la voix hors champ ajoute : « inadapté à la technologie moderne, incapable de lire, il n'obtient rien à l'usine. ». Pendant les scènes d'écoles construites dans le cadre d'une vaste campagne d'alphabétisation, le narrateur commente que «dans les mêmes salles de classe où les enfants apprennent à lire et à écrire et se forment au travail, les adultes se réunissent pour le cours d'alphabétisation ». Les enseignants apparaissent devant ces espaces modernes dont la mise en scène se termine par l'interposition de scènes de pauvreté - dépeintes dans un premier temps - en contrepoint avec les enfants bien alignés à la fin de la vidéo. On peut constater que, selon le film, l'alphabétisation est directement associée aux nouvelles exigences du marché du travail. Ainsi, les images du monde moderne (tracteur, écoles) sont associées à une notion de progrès, tandis que le cheval et la houe sont associés à un retard. 
Image 5 : Extrait du film Éducation et Développement. Le dilemme entre travailler à la campagne et en ville

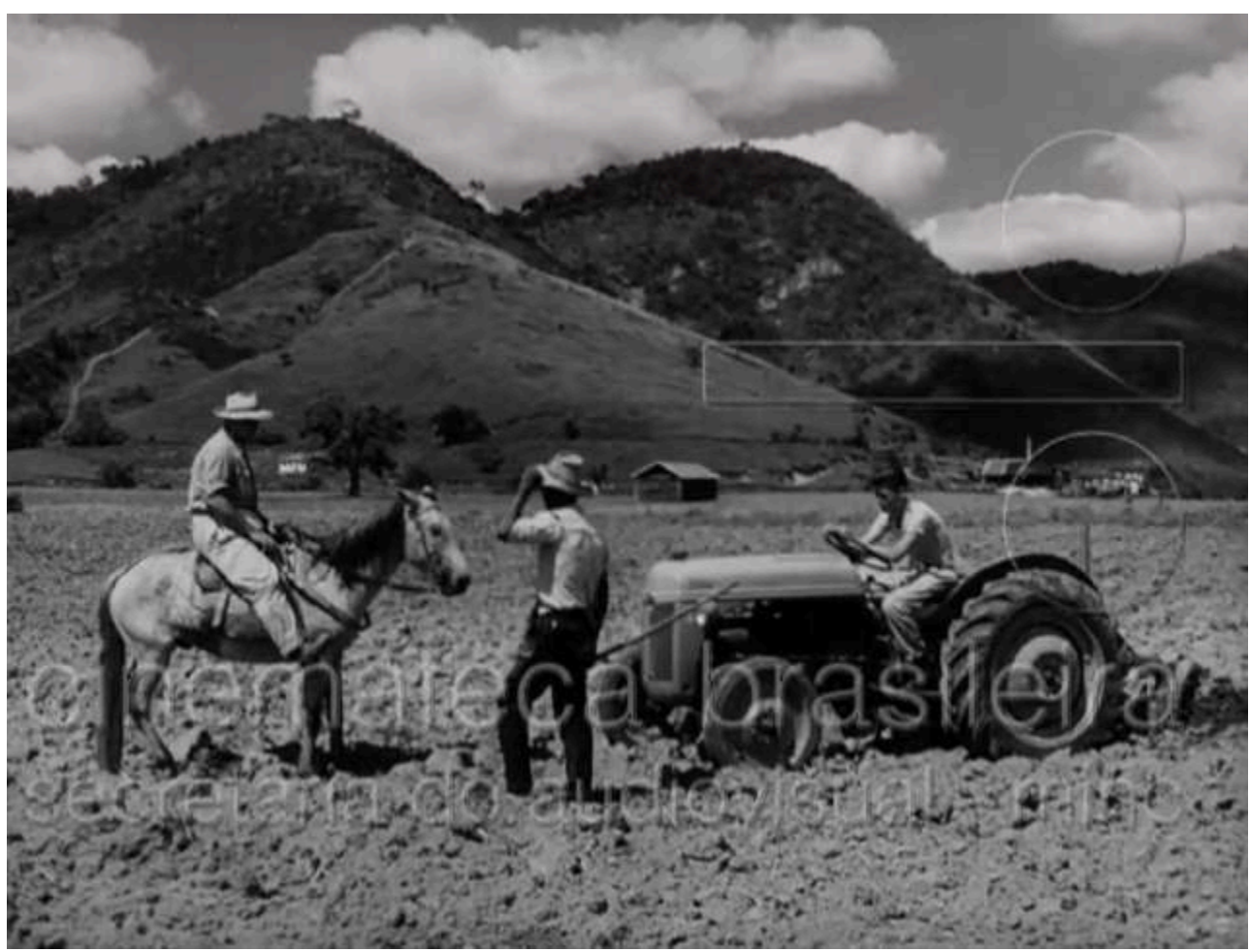

Educação e Desenvolvimento, 1958, Geraldo Santos Pereira, coll. Cinemateca Brasileira.

Les transformations du mode de vie des ouvriers causées par l'exode rural sont également l'un des thèmes présentés dans le film Condamné par le progrès, publié en 1962. La gare de Virajaba, située à Rio de Janeiro, est choisie pour justifier, selon la voix hors champ, les «énormes dégâts » subis par le réseau ferroviaire. Cette vidéo est réalisée à un moment où le pays investit dans les autoroutes comme moyen de mobilité en stimulant la vente de voitures, de camions et de leurs charges, comme le carburant. Le transport de marchandises par la route est la devise de cette production cinématographique de l'INCE. Les graphiques et les animations didactiques (image 6) sont présentés pour mettre en évidence les «branches non rentables » afin que, selon le narrateur, «il n'y ait pas d'autre dilemme : affronter le problème ou gaspiller son énergie dans un effort inutile et financièrement corrosif ». Enfin, des transformations sociales peuvent être observées dans les dernières images qui montrent un cheminot solitaire dans une gare abandonnée (image 7). 
Image 6 : Extrait du film Condamné par le progrès. Utilisation d'animations pour justifier les transformations provoquées par l'exode rural

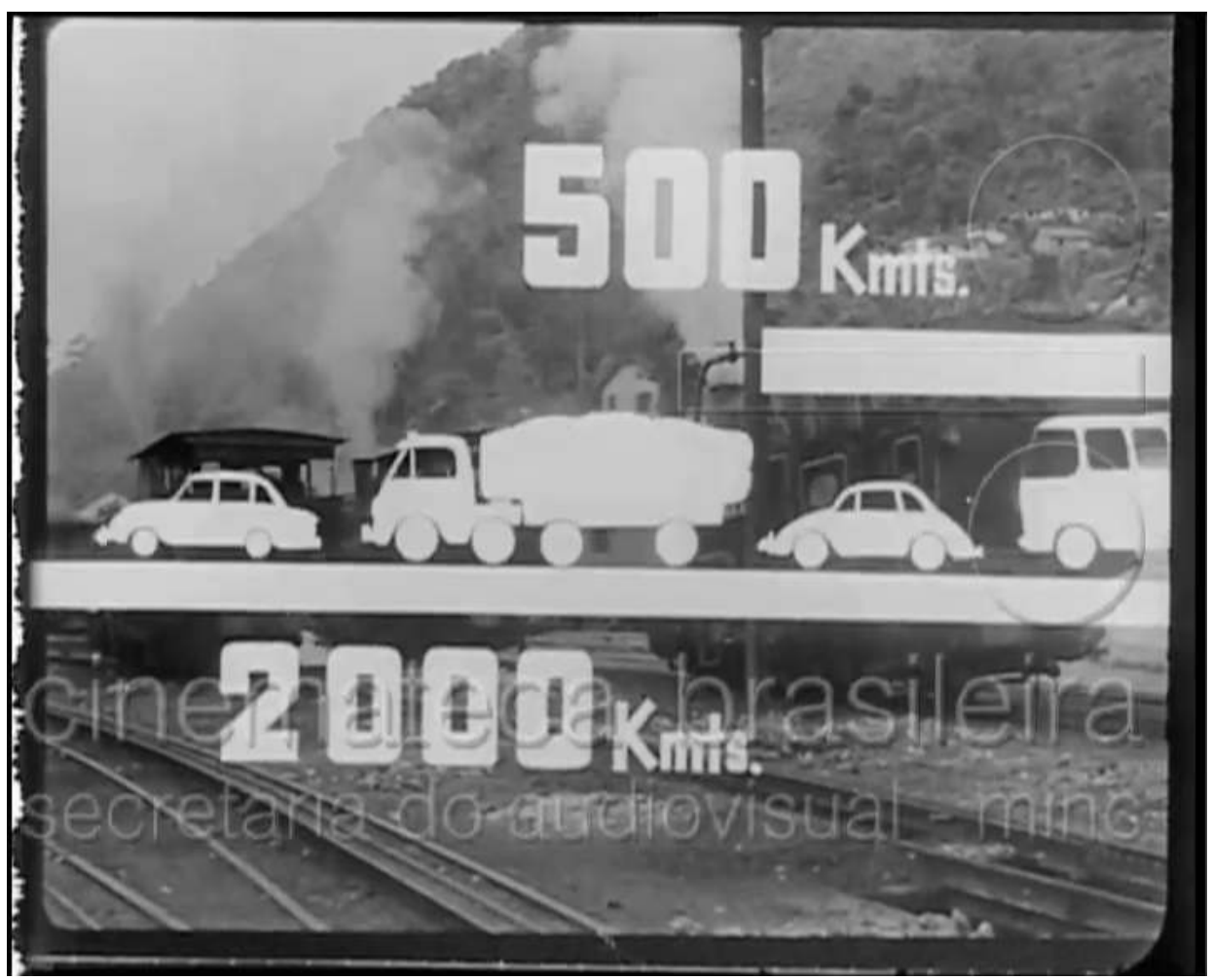

Surimpression d'informations provenant d'aspects routiers sur des images ferroviaires.

Condenados pelo progresso, 1962, Carlos Alberto de Souza Barros, coll. Cinemateca Brasileira.

Image 7 : Extrait du film Condamné par le progrès. Les changements dans le travail et le cheminot solitaire

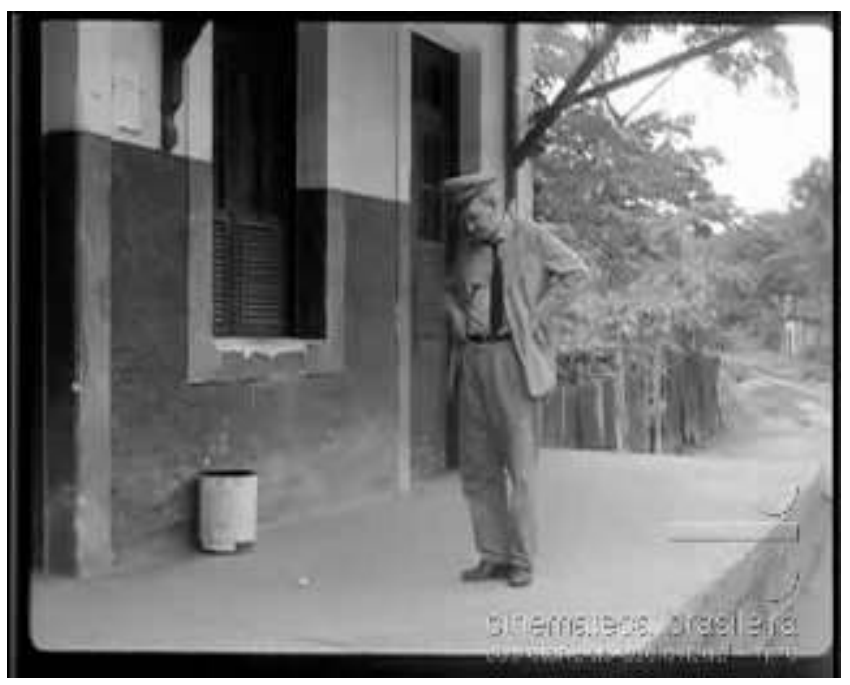

Condenados pelo progresso, 1962, Carlos Alberto de Souza Barros, coll. Cinemateca Brasileira.

21 Toujours dans le domaine du progrès technique, le son d'une machine en mouvement est progressivement remplacé par le son d'une guitare dans l'œuvre audiovisuelle Investir pour progresser, réalisée en 1966. Les images des nouveaux appareils technologiques dans les campagnes et dans les villes corroborent la narration selon 
laquelle « ce n'est qu'avec l'avènement de nouvelles techniques que l'augmentation de la productivité est devenue possible. Les processus de production modernes impliquent des machines. Les machines sont des minéraux transformés par l'industrie qui a besoin d'énergie et de personnel technique spécialisé dont la nourriture dépend de l'agriculture. ». Le son apparaît comme un élément important dans la compréhension de cette œuvre filmique. Enfin, le bruit de la machine en mouvement est accentué lorsqu'un nouveau-né est placé dans un berceau (image 8). Le cri de l'enfant est remplacé par le bruit des engrenages. Selon une telle prémisse, João Bernardo (2009, 329) souligne que «les techniques de gestion, les types de disciplines au travail, les machines, dans leurs remodelages successifs, visent à augmenter le temps de surcharge de travail et à réduire celui du travail nécessaire ». L'ensemble du personnel technique, traité dans ce film comme nécessaire à la croissance du Brésil, semble émerger d'un nouveau-né, privé du contrôle même de sa voix.

Image 8 : Extrait du Investir pour progresser. La voix de l'être humain est remplacée par le bruit du moteur

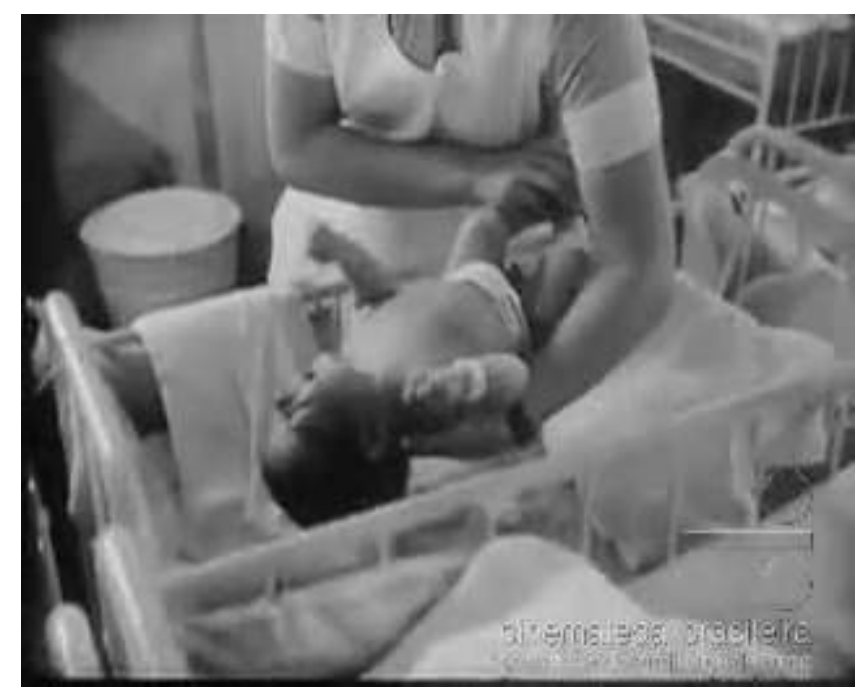

Investir para progredir, 1966, Jacques Deheinzelin, coll. Cinemateca Brasileira.

Comme indiqué dans la première partie de cet article, il existe un grand nombre de films produits par l'INCE qui traitent du thème du travail. Cependant, relevant d'une recherche en cours, les six films présentés témoignent de différentes périodes de la société brésilienne et traversent toutes les administrations de l'Institut national du cinéma éducatif (1936-1966). Le travailleur rural et urbain, les transformations du travail avec l'avènement des nouvelles technologies, la division sexuelle du travail et la formation des travailleurs sont quelques-uns des thèmes abordés dans le matériel filmique.

\section{Conclusion}

L'Institut national du cinéma éducatif est né au Brésil à un moment marqué par l'acuité des discours politiques. Selon le postulat, «l'État n'était pas seulement considéré comme un producteur de biens matériels, mais comme un producteur de discours qui reprenait les éléments clés de l'image de soi des travailleurs et articulait les demandes, 
les valeurs et les traditions de cette classe, en les redimensionnant dans un autre contexte. » (Gomes, 2005, 180). Sur ce point, il convient de noter que le discours est donné par le récit, qui est, à son tour, la narration (sans matérialité) et ce qui narre (matérialité). De cette manière, «l'effet de l'énonciation narrative est [...] produit dans la réalité » (Faye, 2009, 26).

Car c'est face à ce jeu de pouvoir que l'État a trouvé dans l'INCE la possibilité de diffuser les idées des classes dominantes. Dans ce moment marqué par l'urbanisation ajoutée à un taux de croissance des industries au Brésil, "plus que de parler à un public, l'intention était de produire ce public identifié comme la classe ouvrière brésilienne " (Gomes, 2005, 31). Dans cette tentative de valorisation du travail par une harmonisation des conflits du capitalisme, la construction de l'image du travailleur semble se tisser petit à petit dans l'ensemble des œuvres produites par l'INCE.

Ainsi, la construction de l'identité politique de la classe ouvrière ne se fait pas seulement au niveau des relations de production. Dans ce champ de forces, les intérêts corporatistes impliquent plusieurs questions concernant la reconnaissance par l'État de la valeur du travail et les particularités des travailleurs représentés dans les films produits par l'Institut. "Le cinéma est en tout cas un passeur de représentations sociales qui peut contribuer à infléchir ou à enrichir nos façons de penser le monde du travail. » (Jeantet et Savignac, 2012, 60). Ainsi, en s'appuyant sur les particularités du Brésil, l'Institut national de cinéma éducatif a joué un rôle important dans la construction d'un imaginaire national visant à dépasser les critiques et les contestations du système capitaliste. Par conséquent, l'analyse de l'institut revient à se concentrer sur une recherche sur les différents intérêts en jeu dans la société brésilienne qui vont au-delà des simples images animées.

\section{BIBLIOGRAPHIE}

Anderson B. (2008) Comunidades Imaginadas. Reflexões sobre a origem e a difusão do nacionalismo, São Paulo, Cia das Letras.

Bernardet J.-C. (1980) O que é cinema ?, São Paulo, Brasiliense.

Bernardo J. (2009) Economia dos conflitos sociais, São Paulo, Expressão Popular.

Faye J.-P. (2009) Introdução às linguagens totalitárias. Teoria e transformação do relato, São Paulo, Perspectiva.

Gomes A. (2005) A invenção do trabalhismo, Rio de Janeiro, Editora FGV.

Jeantet A. (2012) Cinéma et travail, Travailler, 1 (1), 9-16.

Jeantet A. et Savignac E. (2012) Représentations du monde professionnel et du rapport subjectif au travail dans les films de fiction français contemporains, Travailler, 1 (1), 37-63.

Rangel J. (2010) Humberto Mauro, Recife, Massangana.

Serrano J. et Venâncio F. (1930) Cinema e Educação, Rio de Janeiro, Melhoramentos, vol. XIV. 
Souza C. (1990) Catálogo filmes produzidos pelo INCE, Rio de Janeiro, Fundação do Cinema Brasileiro.

Taillibert C. (1999) L'Institut international du cinématographe éducatif, Paris, L’Harmattan.

Tragtenberg M. (1974) Burocracia e ideologia, São Paulo, Ática.

Vargas G. (1938) O cinema nacional, elemento de aproximação dos habitantes do País. dans A nova política do Brasil, Rio de Janeiro, José Olympio.

\section{NOTES}

1. Cette recherche en cours s'inscrit dans le cadre d'un doctorat qui a débuté en mars 2018 au programme d'études supérieures en sciences sociales de l'Institut de philosophie et de sciences humaines de l'université d'État de Campinas (UNICAMP) au Brésil. La recherche est financée par la Coordination pour l'amélioration du personnel de niveau supérieur (CAPES) et le Comité français d'évaluation de la coopération universitaire avec le Brésil (COFECUB).

2. Serrano et Venâncio, 1930, 137.

3. Ibid.

\section{RÉSUMÉS}

Cet article traite de la manière dont la conception du travail est diffusée par l'Institut national de cinéma éducatif. Reconnu comme le premier organe officiel axé sur l'audiovisuel sur le territoire national, l'INCE a fonctionné de 1936 à 1966 au sein du ministère de l'Éducation et de la Santé publique avec une production totale de 407 œuvres parmi les courts et moyens métrages axés sur l'éducation populaire. Par le biais d'une recherche dans les collections Gustavo Capanema, Edgard Roquette-Pinto et de l'analyse filmique de matériel situé dans la Cinémathèque brésilienne, la recherche vise à décrire comment la représentation du travail dans le cinéma brésilien a été utilisée pour la construction de la nation.

This article addresses how the conception of work is disseminated by the National Institute of Educational Cinema. Recognized as the first official audiovisual body in the country, INCE operated from 1936 to 1966 within the Ministry of Education and Public Health with a total production of 407 works between short and medium-lenght films dedicated to popular education. Through a research in the collections Gustavo Camapena, Roquette-Pinto and the filmic analysis of material located at the Brazilian Cinematheque, the research aims to describe how the representation of work in Brazilian cinema was used for the construction of the nation.

\section{INDEX}

Keywords : social sciences, work, history, cinema, education

Mots-clés : sciences sociales, travail, histoire, cinéma, éducation 


\section{AUTEUR}

\section{RAFAEL BEVERARI}

Rafael Beverari est sociologue et doctorant au programme d'études supérieures en sciences sociales de l'Institut de philosophie et de sciences humaines de l'université d'État de Campinas (UNICAMP) au Brésil. Chercheur du groupe de recherche au Conseil national pour le développement scientifique et technologique (CNPq) Image, Subjectivité et Théorie sociale de l'université fédérale de São Paulo. Chercheur du groupe de travail Anticapitalisme et sociabilités émergentes du Conseil latino-américain des sciences sociales (CLACSO). Membre de l'équipe de doctorants brésiliens de l'accord international CAPES/COFECUB « Le travail au Brésil et en France : sens du changement et changements de sens (2019-2020)», avec stage au Centre de recherches sociologiques et politiques de Paris, équipe Genre, Travail, Mobilités (CRESPPA, GTM, 2020). Il mène actuellement des recherches sur la sociologie, le travail, les luttes sociales et la mémoire. 